\title{
Editorial: Innovative Approaches in Computational Structural Engineering
}

\author{
George C. Tsiatas ${ }^{1}$ and Vagelis Plevris ${ }^{2 *}$ \\ ${ }^{1}$ Department of Mathematics, University of Patras, Rio, Greece, ${ }^{2}$ Department of Civil Engineering and Energy Technology, \\ OsloMet-Oslo Metropolitan University, Oslo, Norway
}

Keywords: structural engineering, innovation, computational, FEM, BEM, meshless methods

\author{
Editorial on the Research Topic
}

\section{Innovative Approaches in Computational Structural Engineering}

Over the last few decades, tremendous development has been made in the field of computing technology, in terms of both computer hardware and software development. As a result of this progress, numerical computation has now become one of the most effective tools for scientists, researchers, and professional engineers around the world. Although this has led to great achievements in computer-based structural engineering, the increasing necessity to solve complex problems in engineering requires the development of new ideas and innovative methods for providing accurate numerical solutions in affordable computing times (Plevris and Tsiatas, 2018).

The Research Topic "Innovative Approaches in Computational Structural Engineering" aims to provide a forum for the presentation and discussion of state-of-the-art innovative developments, concepts, methodologies, and approaches in scientific computation applied to structural engineering. It involves a wide coverage of timely issues on computational structural engineering with a broad range of research and practical applications.

The collection includes both research and advanced applied topics, with particular emphasis

Edited and reviewed by: Georgios Eleftherios Stavroulakis, Technical University of Crete, Greece

*Correspondence: Vagelis Plevris vageli@oslomet.no

Specialty section:

This article was submitted to Computational Methods in Structural

Engineering,

a section of the journal

Frontiers in Built Environment

Received: 05 March 2020 Accepted: 19 March 2020

Published: 08 April 2020

Citation:

Tsiatas GC and Plevris V (2020)

Editorial: Innovative Approaches in Computational Structural Engineering.

Front. Built Environ. 6:39.

doi: 10.3389/fbuil.2020.00039 on innovation in computational structural mechanics and engineering. The contributions come from 58 leading researchers and professionals from 10 countries around the world who are actively involved in the field of computational structural engineering. The papers in the collection cover various relevant scientific areas such as modeling in structural engineering; finite element methods; boundary element methods; static and dynamic analysis of structures; performance-based design; structural optimization; meshless methods; modeling of advanced and innovative materials such as shape memory alloys; non-linear structural analysis; system identification methods; structural stability; GPU computing; earthquake engineering; seismic vulnerability; incremental dynamic analysis; structural damage assessment; smart structures and systems; structural reliability; structural health monitoring and control; optimization; and composite materials, with application to engineering structures.

\section{PAPERS IN THE COLLECTION}

In the 1st paper of the collection, Mitropoulou et al. present a methodology to identify the most appropriate damage index, able to provide a reliable description of the structural damage level. A performance-based design framework is formulated based on this damage index, which is to be used as a design tool for achieving safer and more economic designs. For each damage index under consideration, design optimization problems for structural systems are solved by means of a popular metaheuristic search algorithm. 
Next, Charalampakis and Tsiatas examine three uniaxial phenomenological models, i.e., the Graesser-Cozzarelli model, the Wilde-Gardoni-Fujino model, and the Zhang-Zhu model, which are currently used for the description of a Shape Memory Alloy (SMA) behavior. The examination process reveals several limitations and drawbacks, including the large number of parameters and the unclear effect of specific parameters in the overall response. Based on this analysis, powerful metaheuristics are employed for system identification and a new simple rate-independent model is proposed, which addresses all issues in a unified manner producing excellent fit with the experimental data.

The 3rd paper by Yiotis and Katsikadelis deals with the solution to the vibration problem of cylindrical shell panels using the Meshless Analog Equation Method (MAEM). The method is based on the principle of the analog equation, converting the original equations into three substitute ones, two Poisson's and one biharmonic, which are solved using a meshless method. The use of integrated MultiquadricRadial Basis Functions (MQ-RBFs) to approximate the fictitious sources allows the approximation of the solutions sought by new RBFs, which accurately approximate both the solution and its derivatives. Several shell panels are analyzed using the method, and the numerical results demonstrate its efficiency and accuracy.

Tsalkatidis et al. study the mechanical behavior of bolted hybrid connections that consist of a square hollow steel column (SHS) and a glulam timber beam. A reference model is constructed and verified by comparison to experimental and numerical data from the literature. Several parameters that affect the response of the connection are modified in order to investigate and quantify their effect, resulting in seven different case studies. A proposed optimal configuration of the hybrid connection is investigated and presented.

In the next paper, Tsiatas et al. present a new layered approach to the non-linear analysis of initially straight EulerBernoulli rectangular beams by the Boundary Element Method (BEM). The formulation of the problem is based on the displacements, and the equations of equilibrium, derived from the principle of minimum total potential energy, being coupled and non-linear. The beam is discretized in both the longitudinal direction and the cross-sectional plane, and the governing differential equations are solved using the Analog Equation Method (AEM) in conjunction with an iterative numerical process. Various representative examples are examined that not only take geometrical nonlinearity into account but material non-linearity as well. The reliability and effectiveness of the proposed method are validated by comparing the results obtained with those presented in the literature or derived by other Finite Element (FE) models.

Arailopoulos et al. propose an integrated reverse engineering methodology for a large-scale fully operational steam turbine rotor, addressing issues that include developing the CAD and FE model of the structure, as well as the applicability of model updating techniques based on experimental modal analysis procedures. The FE model of the turbine was developed using tetrahedral solid elements resulting in 55 million DOFs. Experimentally identified modal modes and modal frequencies were compared to the FE model predictions, and CMA-ES optimization was used to fine-tune the material parameters, such as modulus of elasticity and density. A simplified FE model was also developed and used for the turbine rotordynamic analysis.

In the 7th paper, Mascolo et al. developed a FE model of the Moderate Rotation Theory (MRT) of laminated composite beams. The proposed model describes laminated composite beams with arbitrary curvature of the beam axis taking into account shear deformation, warping effects, and in-plane and out-of-plane instability. The stability analysis is performed through a path-following procedure and a bordering algorithm. Several numerical results are presented and comparisons are made with classical beam theories and other theories available in the relevant literature.

Jena and Chakraverty investigate the free vibration of the variable cross-section (non-uniform) Single-Layered Graphene Nano-Ribbons (SLGNRs) by using the Differential Quadrature Method (DQM). The Euler-Bernoulli beam theory is considered in conjunction with Eringen's non-local elasticity theory. A convergence study is carried out to illustrate the efficiency of the method and the obtained results are validated with known results.

In the paper by Molina-Moya et al, a parallel iterative solver based on the Generalized Minimum Residual Method (GMRES) with complex-valued coefficients is explored, with applications to BEM. The proposed approach does not require modifications in the main program, which computes the system matrix and the right vector, allowing the use of standard BEM codes, with the solver task being transferred to the Graphic Processing Unit (GPU). The CUDA programming language is used, exploiting the particular architecture of the GPU device for complex-valued systems. The parallelized GMRES solver shows reductions in computing times when compared with its CPU implementation.

In the 10th paper, Panagiotopoulos et al. present a unified methodology to solve problems of frictionless unilateral contact as well as adhesive contact between linear elastic solids. The methodology is based on energetic principles and is applied to a minimization problem of the total potential energy. To solve the quadratic problem, two algorithms are developed, which are both variants of the well-known conjugate gradient algorithm. Both symmetric and nonsymmetric formulations of this matrix are presented and discussed, showing that the non-symmetric formulation provides more accurate results. The framework is found to be very useful in cases where dissipative mechanisms also exist on the boundaries or common interfaces of elastic bodies.

Blasi et al. present a work aimed at developing a hybrid approach to consider the effect of concrete cracking on the hysteretic response of RC frames. The smeared cracking approach is employed, while discrete cracking 
surfaces are included in the geometrical model where the interface behavior is defined by the combination of contact and cohesive elements. The proposed approach is adopted in ABAQUS to simulate an experimental test on a double cantilever column for the calibration of the numerical model. Numerical and experimental results are compared in terms of hysteretic force-displacement behavior and cumulative dissipated energy, to assess the reliability of the proposed model in simulating the energy dissipation capacity of RC members subjected to lateral cyclic loading.

In the 12th paper by Zhao et al., three different strategies are proposed to identify the general hysteretic behavior of a typical shear structure subjected to external excitations. Different case studies are presented to analyze the dynamic responses of a time-varying shear structural system with the early version of the Bouc-Wen-Baber-Noori (BWBN) hysteresis model. Various computational techniques are utilized. The results show that Intelligent Parameter Varying (IPV) performs superior computational efficiency and system identification accuracy in comparison to Genetic algorithm (GA), and Transitional Markov Chain Monte Carlo (TMCMC) approaches.

The subject of the 13th work of the collection, by Giordano et al., is Sant' Agostino Sanctuary in Offida, central Italy. The village and the sanctuary itself was severely damaged by the seismic events of August 2016. A finite element model using non-linear static analysis (Pushover) is employed to estimate the vulnerability of the sanctuary and its dynamic response, considering the nonlinear behavior of masonry with a homogenized material and a smeared cracking and crushing constitutive law. The structure is shown to be prone to massive damage leading to collapse.

Repapis and Zeris investigate the vulnerability assessment of non-conforming infilled RC structures of the 1960s and 1970s. The seismic performance of typical bare and infilled structures was evaluated. The results from Static Pushover analyses are extended with Incremental Dynamic Analysis predictions using a large number of recorded base excitations from recent destructive earthquakes in Greece and internationally. The analysis shows that fully or partially infilled RC frames can perform well, while frames with an open floor usually have the worst performance due to the formation of an unintentional soft story.

In the 15th paper, Juárez-Luna and Caballero-Garatachea study the nonlinear behavior of RC circular, elliptic, and triangular isolated slabs using computational mechanics. The concrete was modeled with a damage model which includes softening, while the behavior of the reinforcing steel was simulated with a $1 \mathrm{D}$ bilinear plasticity model. Validation was done by comparing the computed numerical results with the experimental results reported in the literature. The coefficient method is proposed for its simplicity to calculate design bending moments in slabs with arbitrary geometries.
Soroushian and Farahani apply a technique proposed in 2008 by Soroushian (2008) to the FE analysis of assemblies of beam-columns subjected to continuous static loads. These loads may be computationally expensive especially when they are densely digitized. It is shown that the adapted technique might considerably reduce the computational cost with only a negligible change in accuracy. Furthermore, the good performance of the adapted technique is demonstrated from different points of view and is compared with the performance of the technique in time integration analysis.

In the 17th paper, Marakakis et al. review the current state of the art in shunt piezoelectric systems for noise and vibration control. In fact, resonant shunts have proved to be very efficient and stable for the reduction of vibration on smart piezoelectric structures, such as beams and plates. From the numerous applications which were reviewed it is evident that shunt piezoelectric systems can be very effective for several different purposes such as control of vibrations on hard drives, noise reduction on acoustic applications, improvement of image quality and scan rate of tapping mode Atomic Force Microscopy.

In the last paper of the collection, Papazafeiropoulos et al. propose a novel spectra-matching framework, which employs a linear combination of raw ground motion records to generate artificial acceleration time histories for matching a target spectrum taking not only the acceleration into account, but also the seismic input energy equivalent velocity. The procedure of selection and scaling of the suite of ground motion records to fit a given target spectrum is formulated by means of an optimization problem. The optimization results have shown that there is good agreement between the target and optimized spectra for the different matching scenarios examined, regardless of the nature of the target spectrum.

In closing this editorial, we would like to thank the contributing authors, the reviewers, the staff at Frontiers and all the other people we have worked closely with in trying to make this Research Topic a success. Once again, it has been proven that computational methods are at the forefront of real-world applications. Especially in the structural engineering discipline, the applications are numerous and cover a wide range of topics. Although very powerful capabilities are now available for the simulation and analysis of structures, there are still many exciting research challenges. The field of computational structural engineering will continue to grow and increasingly contribute to technological development in the future, influencing and enriching the engineering profession and our lives in general.

\section{AUTHOR CONTRIBUTIONS}

GT and VP handled manuscripts and edited the Research Topic. Both authors have made a substantial, direct and intellectual contribution to the work, and approved it for publication. 


\section{REFERENCES}

Plevris, V., and Tsiatas, G. (2018). Computational structural engineering: past achievements and future challenges. Front. Built Environ. 4:21. doi: 10.3389/fbuil.2018. 00021

Soroushian, A. (2008). A technique for time integration analysis with steps larger than the excitation steps. Commun. Numer. Methods Eng. 24, 2087-2111. doi: 10.1002/ cnm. 1097
Conflict of Interest: The authors declare that the research was conducted in the absence of any commercial or financial relationships that could be construed as a potential conflict of interest.

Copyright (c) 2020 Tsiatas and Plevris. This is an open-access article distributed under the terms of the Creative Commons Attribution License (CC BY). The use, distribution or reproduction in other forums is permitted, provided the original author(s) and the copyright owner(s) are credited and that the original publication in this journal is cited, in accordance with accepted academic practice. No use, distribution or reproduction is permitted which does not comply with these terms. 